\title{
Realistic many-body models for Manganese Monoxide under pressure
}

\author{
Jan M. Tomczak, ${ }^{1,2}$ T. Miyake, ${ }^{1,2}$ and F. Aryasetiawan ${ }^{3,1,2}$ \\ ${ }^{1}$ Research Institute for Computational Sciences, AIST, Tsukuba, 305-8568 Japan \\ ${ }^{2} J a p a n$ Science and Technology Agency, CREST \\ ${ }^{3}$ Graduate School of Advanced Integration Science, Chiba University, Chiba, 263-8522 Japan
}

\begin{abstract}
In materials like transition metals oxides where electronic Coulomb correlations impede a description in terms of standard band-theories, the application of genuine many-body techniques is inevitable. Interfacing the realism of density-functional based methods with the virtues of Hubbardlike Hamiltonians, requires the joint $a b$ initio construction of transfer integrals and interaction matrix elements (like the Hubbard U) in a localized basis set. In this work, we employ the scheme of maximally localized Wannier functions and the constrained random phase approximation to create effective low-energy models for Manganese monoxide, and track their evolution under external pressure. We find that in the low pressure antiferromagnetic phase, the compression results in an increase of the bare Coulomb interaction for specific orbitals. As we rationalized in recent model considerations [Phys.Rev.B79, 235133(2009)], this seemingly counter-intuitive behavior is a consequence of the delocalization of the respective Wannier functions. The change of screening processes does not alter this tendency, and thus, the screened on-site component of the interaction - the Hubbard $U$ of the effective low-energy system - increases with pressure as well. The orbital anisotropy of the effects originates from the orientation of the orbitals vis-à-vis the deformation of the unit-cell. Within the high pressure paramagnetic phase, on the other hand, we find the significant increase of the Hubbard $U$ is insensitive to the orbital orientation and almost exclusively owing to a substantial weakening of screening channels upon compression.
\end{abstract}

PACS numbers:

\section{INTRODUCTION}

\section{A. Realistic models for correlated materials}

While standard band structure methods, like density functional theory (DFT) in the local spin density approximation (LSDA), ${ }^{1,2}$ in principle allow for a treatment of the realistic complexity of materials arising from orbital, spin and structural degrees of freedom, the failure of these techniques in the presence of strong electronic correlations is well documented. ${ }^{3}$ Many-body techniques, on the other hand, while potentially treating correlation effects with accuracy, often lack the realism to account for the electronic structure of a material from a firstprinciples basis.

Hence, when it comes to the $a b$ initio description of correlated matter, the joining of these two fields is of paramount importance. Often, this combining amounts to constructing effective, yet realistic, low energy models, in the spirit of the Hubbard or Anderson model. These consist of a one-particle part, extracted from the KohnSham band structure, and two-particle interaction terms. There are different paradigms for choosing the matrix elements of the latter. Sometimes, these values are treated as mere empirical parameters, adjusted to correctly reproduce some experimental finding. However, there exist first-principle methods, among which the most popular are the constrained LDA technique, ${ }^{4}$ and the constrained random phase approximation (cRPA). ${ }^{5}$

In this article we are chiefly concerned with the evolution of interaction matrix elements under pressure within a realistic setup. While the influence of pressure onto structures and band-structures has been widely studied on the DFT level, changes in the interaction have received much less attention. Yet, the pronounced sensitivity of correlated matter on external parameters ${ }^{6}$ heralds the need for an accurate treatment of all ingredients to the electronic structure. For the $\mathrm{LDA}+\mathrm{U}^{7}$ technique, this issue has been investigated within a linear response based approach ${ }^{8-10}$, and the reproduction of crystal and electronic structures, as well as phase stabilities were indeed found to require the description of pressure induced changes in the Hubbard $U$.

Here, we are interested in setting the stage for manybody methods that go beyond density functional methods. In this vein, a particularly versatile technique is the use of maximally localized Wannier functions ${ }^{11,12}$ within the cRPA setup, ${ }^{13,14}$ which is the method of choice of this article. In this formalism it is possible to turn off precisely those screening channels that are to be left to the solution of the effective model. ${ }^{5,67}$ Moreover, the use of many-body techniques often requires the use of localized basis sets, and the Wannier construction is geared at exactly this. While the Wannier-cRPA technique has been already applied to several systems, such as $3 \mathrm{~d}$ transition metals, ${ }^{13}$ the perovskite $\mathrm{SrVO}_{3},{ }^{13}$ and oxypnictides $^{15,16}$, the evolution of the respective Coulomb interaction matrix elements under external stimuli such as pressure have not been addressed in the realistic case. In recent model considerations, ${ }^{14}$ we established generic behaviors of maximally localized Wannier functions and interaction matrix elements under pressure for simple setups. We rationalized that as a consequence of the delocalization of Wannier functions under compression, not only do transfer integrals increase, but also - counter- 
intuitively - the matrix elements of the bare Coulomb interaction augment. In this paper, we extent this work to a more complex scenario by applying the fully $a b i n i$ tio Wannier-cRPA formalism to manganese monoxide, $\mathrm{MnO}$, under pressure. In particular, we monitor the evolution of transfer integrals, on-site matrix elements of the bare Coulomb interaction, and of the partially screened Coulomb interaction (the Hubbard U, and the Hund J), for different realistic low-energy systems. We will distinguish between pressure induced changes related to the construction of the sub-system, and changes in the screening strength as provided by the band-structure and transition matrix elements in the polarization. We find that while the former mainly determine the behavior of the interactions in the low temperature, low pressure anti-ferromagnetic phase of $\mathrm{MnO}$, changes in the Hubbard $U$ of the high pressure paramagnetic phase are dominated by screening related effects.

\section{B. Manganese monoxide}

\section{Approaches to the crystal and electronic structure}

Concerning the failures of the LDA in the realm of correlated materials, the focus material of this work, manganese monoxide, $\mathrm{MnO}$, is rather well behaved at first glance : Below $118 \mathrm{~K}$ the system is antiferromagnetic and due to the fact that the Mn3d orbitals are halffilled, spin-dependent band-structure calculations ${ }^{17-24}$ do indeed yield an insulator. However, above the Néel temperature, which is much smaller than the experimental charge gap of $3.9 \mathrm{eV},{ }^{25} \mathrm{MnO}$ is paramagnetic, and believed to be a Mott insulator. A straight forward bandstructure calculation in the paramagnetic phase, however, yields a metal.

A genuine feature of correlated materials is their sensitivity to external stimuli, leading to rich phase-diagrams with regimes of different spin or orbital orders, and metalinsulator transitions. ${ }^{6} \mathrm{MnO}$ is no exception and both experiments and theory helped to elucidate the phases of manganese monoxide as a function of temperature versus pressure. See Fig. 1 in Ref. ${ }^{26}$ for a graphical depiction of compiled results.

The paramagnetic insulator $\mathrm{MnO}$, which is of rocksalt structure (B1), contracts below the Néel temperature along the $\langle 111\rangle$ direction, resulting in a rhombohedral distorted phase (dB1). $\mathrm{MnO}$ has been the prototype material for the discovery and description of the superexchange mechanism. ${ }^{27-29}$ It was rationalized by Terakura et al. ${ }^{17}$ that this strong manganese superexchange via the oxygen orbitals 27,28 is responsible for a spin oder that is of AF II type, ${ }^{30-32}$ with alternating spins along the distortion axis. With increasing pressure, the Néel temperature rises $^{33,34}$ and reaches room temperature $(300 \mathrm{~K})$ at $30 \mathrm{GPa} .{ }^{26}$ It has been shown that beyond the volume compression, it is the rhombohedral distortion that stabilizes the AF II order under pressure with respect to other spin orientations. ${ }^{18}$ At constant temperature $(300 \mathrm{~K})$, a further transition occurs at $90 \mathrm{GPa},{ }^{26,35,36}$ when the system becomes, again, a paramagnetic insulator, yet of NiAs (B8) structure. ${ }^{26}$ Notably, the high pressure B8 structure was predicted by DFT calculations in the generalized gradient approximation (GGA). ${ }^{18}$ At still higher pressure, the system undergoes an iso-structural Mott insulator to metal transition at $105 \mathrm{GPa},{ }^{26}$ signaled by a volume collapse of $6.6 \%,{ }^{26}$ metallic lustre, ${ }^{36}$ an increase of reflectance, ${ }^{37,38}$ a resistance decrease of several orders of magnitude ${ }^{39}$ and a quenching of the magnetic moment. ${ }^{26}$ The transition was anticipated, from theoretical considerations $^{40}$ and $a b$ initio calculations. ${ }^{19}$ Yet, both works forward different mechanism : In Ref. ${ }^{40}$ a pressure induced increase in the crystal-field was invoked to explain the metalization, while in Ref. ${ }^{19}$, in which the rhombohedral distortion is neglected, a growing band-width was seen at the origin of the transition. We will come back to this in the discussion of our results, which favor the first point of view.

On the theory side, numerous works that aim at reproducing the described phase-diagram from first principles resort to improvements within the density functional formalism. Among these are the generalized gradient approximation (GGA), ${ }^{18-24}$ selfconsistent Hartree, ${ }^{41}$ LDA/GGA $+\mathrm{U}^{18,20-23,42,42}$ selfinteraction corrected (SIC) LSDA. ${ }^{43,44}$ Also applied were various hybrid functional ${ }^{45}$ based approaches ${ }^{20,21,23,24}$.

Although relying on the coincidence of a half-filled dshell and the spin order for insulating results, it is due to the fact that (unlike for the other transition metal monoxides) the $3 d$ electrons are not falsely itinerant, that the above methods succeed in yielding at times reasonable structures, lattice constants, bulk moduli, magnetic moments, and sometimes even values for the charge gap (see especially the SIC and hybrid functional references from above). Results have been obtained also within the GW approximation. ${ }^{46-48}$ While pioneering work ${ }^{49}$ still had to resort to numerical simplifications, the increase in computer performance nowadays even allows the application of self-consistency schemes to $\mathrm{MnO},{ }^{50}$ which considerably improve on the gap value of the antiferromagnetic phase with respect to LDA calculations.

Yet, despite these successes, it is evidently desirable to have a many-body technique that works in a wider context, capturing correlated metals, ordered and Mott insulators. In this vein, the probably most generally applicable approach nowadays is LDA+DMFT, ${ }^{51}$ where a Hubbard Hamiltonian consisting of a one-particle part stemming from the LDA and an interaction part of Hubbard-Hund type is solved within dynamical mean field theory (DMFT). ${ }^{52,68}$ While DFT methods and their generalizations, including $\mathrm{LDA}+\mathrm{U},{ }^{7}$ cannot address finite-temperature insulators without broken symmetries, LDA+DMFT is in principle capable to deal with all the different phases. Concerning $\mathrm{MnO}$, the technique has first been used for the calculation of phonon dispersions. ${ }^{53}$ Recently LDA+DMFT was applied by 
Kuneš et $a l .54$ to study the isostructural Mott insulator to metal transition witnessed at $105 \mathrm{GPa}(300 \mathrm{~K})$. The results reproduced quite accurately all experimental findings, in particular the critical pressure and the joint collapse of volume and magnetic moment. ${ }^{54}$

Often it is criticized that $\mathrm{LDA}+U$ and $\mathrm{LDA}+\mathrm{DMFT}$ calculations are not fully $a b$ initio since the interactions are treated as "parameters" that are in a sense tuneable. Indeed in the above LDA+DMFT work, ${ }^{54}$ the Hubbard $U$ and the Hund $J$ were chosen to be constant as a function of pressure. Although adjustable parameters are to be preferred over artificially broken phases, it is true that especially in transition metal monoxides there are several competing energy scales ${ }^{55}$ so that the values of the interactions have to be known with greater precision than in other compounds. Among those relevant energies are : the crystal field splitting, that measures the degree of non-degeneracy of the centers of gravity of the d orbitals, e.g. $\Delta_{c f}=\bar{\epsilon}_{e_{g}}-\bar{\epsilon}_{t_{2 g}}$ for an octahedral coordination, the charge transfer energy $\Delta_{c t}=\bar{\epsilon}_{t_{2 g}}-\bar{\epsilon}_{2 p}$ here defined between the $\mathrm{O} 2 p$ and the $\mathrm{Mn} t_{2 g}$, the transfer integrals (hoppings) $t_{t_{2 g}}, t_{e_{g}}$, the hybridizations $t_{p d}$, and the onsite Coulomb interaction $U$ of the d orbitals.

\section{Earlier works on the Hubbard $U$}

In the pioneering work of Anisimov et al. ${ }^{7}$ that introduced the LDA $+U$ formalism, the constrained LDA technique was applied to $\mathrm{MnO}$ at zero pressure and a value $U=6.9 \mathrm{eV}$ was found. The Hubbard $U$ is thus of the same order as the charge transfer energy $\Delta_{c t}$ (see below). Therewith $\mathrm{MnO}$ is in the intermediate regime between a charge transfer and a Mott-Hubbard insulator ${ }^{55}$ and changes in $U$ might considerably alter the many-body spectra and their interpretation.

An empirical technique that was applied to $\mathrm{MnO}$ is the fitting of configuration interaction (CI) cluster calculations to photoemission experiments. Although of course depending on the details of the chosen cluster, we cite the values $U=7.5 \mathrm{eV}, \Delta_{c t}=7.0 \mathrm{eV}$ of Fujimori et al. ${ }^{56}$ and $U=8.5 \mathrm{eV}, \Delta_{c t}=8.8 \mathrm{eV}$ of van Elp et al. ${ }^{25}$ Still, CI cluster calculations cannot account for delocalized bands. As a consequence, the struggle of d electrons between localized and itinerant behavior and the mixing with the more extended $\mathrm{O} 2 p$ and Mn4s orbtials is biased.

Using a semi-empirical Anderson (d-) impurity Hamiltonian, Zaanen and Sawatzky ${ }^{29}$ estimated $\Delta$ and $U$ to be both around $9 \mathrm{eV}$, and found accurate values also for the Néel temperature. An Anderson Hamiltonian was recently also applied to results from resonant inelastic $\mathrm{x}$-ray scattering (RIXS) spectra $^{57}$ : the parameters of a single manganese d impurity model were adjusted to yield $U=7.2 \mathrm{eV}$ and $\Delta_{c t}=6.5 \mathrm{eV}$. In this approach, the delocalized character of the oxygen and Mn4s orbitals is better captured than within CI based methods. Indeed, this is a step toward LDA+DMFT calculations, albeit without obeying the self-consistency condition that re- lates the manganese impurity with the periodic solid.

The Hubbard $U$ can also be extracted from GW calculations. Indeed the RPA technique is a crucial ingredient to the GW approximation. Pioneering model GW calculations suggested a value of $U=8.0 \mathrm{eV}^{49}$ for the antiferromagnetic phase, albeit only empirically extracted from the energy difference between occupied and unoccupied $e_{g}$ and $t_{2 g}$ states.

\section{METHOD}

The method of using the Wannier orbital construction in conjunction with the constrained RPA has been presented in detail in Ref. ${ }^{13}$. Here, we shall just give a brief summary as is necessary for understanding our results.

\section{A. Band-structure}

In this work, we employ the LSDA in the full-potential (FP) $\mathrm{LMTO}^{58}$ realization and use experimental structures and lattice parameters as provided by Yoo et al. ${ }^{26}$ at $300 \mathrm{~K}^{69}$ and variable pressure. We use up to 4096 reducible Brillouin zone points, the LMTO basis set includes orbitals up to $l=4(3)$ for $\mathrm{Mn}(\mathrm{O})$, and we use local orbitals $^{58}$ to incorporate semi-core and high-lying states.

\section{B. Maximally localized Wannier functions}

The one-particle part of the effective low energy systems is extracted from the (LMTO) band-structure calculation by the construction of a Wannier basis ${ }^{59}$ of the desired subset of orbitals. This procedure not being unique, ${ }^{59}$ we choose to use Wannier functions that are maximally localized. ${ }^{11,12}$ While the initial Hilbert space is spanned by all Kohn-Sham wave functions, $\mathcal{H}=\operatorname{span}\left\{\psi_{\mathbf{k} \alpha}^{\mathrm{KS}}\right\}$, for the aim of constructing effective low energy models necessitates the choosing of a subspace, $\mathcal{H}^{\text {low }} \subset \mathcal{H}$, made up by only selective Kohn-Sham orbitals. The Wannier functions are then deduced from the latter. Complications occur if the bands of the orbitals that constitute the desired sub Hilbert space are entangled with high energy bands. For details on how then to construct maximally localized Wannier functions see $^{12}$, and for a comparison with other Wannier function schemes, for instants the approach in which the on-site screened Coulomb interaction is maximized, see e.g. the Refs. ${ }^{13,14,60}$.

\section{C. constrained RPA}

The matrix elements of the bare Coulomb interaction $v\left(\mathbf{r}, \mathbf{r}^{\prime}\right)=\frac{e^{2}}{4 \pi \epsilon_{0}} 1 /\left|\mathbf{r}-\mathbf{r}^{\prime}\right|$ in the Wannier basis $\chi_{\mathbf{R} \alpha}^{\mathrm{W} *}(\mathbf{r})$ are 


\begin{tabular}{c||c|c} 
setup & low energy subspace $\mathcal{H}^{\text {low }}$ & polarization $P_{\text {low }}$ \\
\hline \hline $\mathrm{d}$ & $\operatorname{span}\left\{\psi_{\mathbf{k} \alpha}^{K S} \mid \alpha=\mathrm{Mn} 3 d\right\}$ & $P_{d}$ \\
$\mathrm{pd}$ & $\operatorname{span}\left\{\psi_{\mathbf{k} \alpha}^{K S} \mid \alpha=\mathrm{Mn} 3 d, \mathrm{O} 2 p\right\}$ & $P_{d}+P_{p}+P_{p d}+P_{d p}$ \\
$\mathrm{pd}+\mathrm{p}$ & $\operatorname{span}\left\{\psi_{\mathbf{k} \alpha}^{K S} \mid \alpha=\mathrm{Mn} 3 d, \mathrm{O} 2 p\right\}$ & $P_{d}$
\end{tabular}

TABLE I: Summary of the low-energy setups that are used : Shown are the choices for the construction of the low energy subspace, and the polarization. See text for details.

given by

$$
\begin{aligned}
& V_{\mathbf{R}, \mathbf{R}^{\prime}}^{\alpha \beta \alpha^{\prime} \beta^{\prime}}=\frac{e^{2}}{4 \pi \epsilon_{0}} \times \\
& \int d^{3} r d^{3} r^{\prime} \chi_{\mathbf{R} \alpha}^{\mathrm{W} *}(\mathbf{r}) \chi_{\mathbf{R} \beta}^{\mathrm{W}}(\mathbf{r}) \frac{1}{\left|\mathbf{r}-\mathbf{r}^{\prime}\right|} \chi_{\mathbf{R}^{\prime} \alpha^{\prime}}^{\mathrm{W} *}\left(\mathbf{r}^{\prime}\right) \chi_{\mathbf{R}^{\prime} \beta^{\prime}}^{\mathrm{W}}\left(\mathbf{r}^{\prime}\right)
\end{aligned}
$$

From the latter, partially screened interaction matrix elements are obtained using the cRPA technique. ${ }^{5,13}$ Using the Kohn-Sham orbitals, the total RPA polarization can be expressed by

$$
\begin{aligned}
& P\left(\mathbf{r}, \mathbf{r}^{\prime}, \omega\right)= \\
& \sum_{\text {spin }} \sum_{\mathbf{k} n}^{\text {occ }} \sum_{\mathbf{k}^{\prime} n^{\prime}}^{\text {unocc }} \psi_{\mathbf{k} n}^{\mathrm{KS} *}(\mathbf{r}) \psi_{\mathbf{k}^{\prime} n^{\prime}}^{\mathrm{KS}}(\mathbf{r}) \psi_{\mathbf{k}^{\prime} n^{\prime}}^{\mathrm{KS} *}\left(\mathbf{r}^{\prime}\right) \psi_{\mathbf{k} n}^{\mathrm{KS}}\left(\mathbf{r}^{\prime}\right) \\
& \times\left\{\frac{1}{\omega-\epsilon_{\mathbf{k}^{\prime} n^{\prime}}+\epsilon_{\mathbf{k} n}+\imath 0^{+}}-\frac{1}{\omega+\epsilon_{\mathbf{k}^{\prime} n^{\prime}}-\epsilon_{\mathbf{k} n}-\imath 0^{+}}\right\}
\end{aligned}
$$

Its matrix elements within the Wannier basis set are given analogous to the above bare interaction. The strength of screening channels can thus be influenced by the overlap integrals (matrix elements) of the wave functions, and by the energy difference of the Kohn-Sham excitations that appear in the denominators.

From the form of the fully screened interaction, $W(\omega)=[1-P(\omega) v]^{-1} v$ (that also appears in the GW formalism ${ }^{46-48}$ ) it can be shown, ${ }^{5}$ that the screening contributions are actually additive. Indeed one can split the polarization, $P=P_{l o w}+P_{r}$, into the transitions within a low-energy orbital subspace, $P_{\text {low }}$ of $\mathcal{H}^{\text {low }}$, and the rest $P_{r}=P_{\text {high-high }}+P_{\text {high-low }}+P_{\text {low-high }}$, where the latter includes, both, transitions within the high-energy subspace, and between the two subspaces. Then the fully screened interaction can be written recursively, i.e. $W=\left[1-P_{\text {low }} W_{r}^{\text {low }}\right]^{-1} W_{r}^{\text {low }}$ where

$$
W_{r}^{\text {low }}=\frac{v}{1-P_{r} v}
$$

is the Coulomb interaction when all screening processes that do not involve transitions within the low-energy orbital subspace have been accounted for ${ }^{70}$.

For our compound, $\mathrm{MnO}$, we will consider three different setups, that are also summarized in Tab. I.

- In the "d-setup", a many body model is obtained from maximally localized Wannier functions that are constructed for the space of the Mn3d KohnSham (KS) orbitals, $\psi_{\mathbf{k}, 3 d}^{K S}$, and from a partially screened interaction $W_{r}^{d}$ that is given by constraining the polarization to transitions that are not fully within the d-subspace ${ }^{71,72}$.

- $\mathrm{MnO}$ being close to the charge transfer regime, it is sensible to include the oxygen $2 p$ orbitals for a realistic many-body treatment. In such a "pdsetup" one constructs a Wannier basis for the space of Mn3d and O2p Kohn-Sham wavefunctions, and chooses $P_{r}=P-P_{d}-P_{p}-P_{p d}-P_{d p}$.

- Yet, often, a Hubbard $U$ is only put on the most correlated orbitals, here the Mn3d ones. In that case, while still constructing a full pd Wannier basis, one should allow for oxygen screening in the RPA (i.e. $P_{r}=P-P_{d}$ ), since those channels are blocked when the interactions between the $\mathrm{p}$ and $\mathrm{d}$ orbitals are omitted. Therewith, $W_{r}\left(\mathbf{r}, \mathbf{r}^{\prime}\right)$ of the current setup and the pd one are the same, yet their matrix-element will differ due to the Wannier different basis.

As an outlook, we like to mention that in the described procedure to generate the effective many-body problem, couplings in the interaction between the low energy subspace and the other orbital degrees of freedom are discarded - an approximation that might prove insufficient if the subspace is chosen too restrictively. A framework that separates the Hilbert space, while retaining the influence of self-energy effects between subspaces has been recently proposed in Ref. ${ }^{61}$.

\section{Wannier functions and Coulomb matrix elements under pressure}

Before applying the above techniques to our compound of interest, we shall first discuss what circumstances will influence the Wannier functions and the interaction values. We can distinguish three conceptually different mechanisms : The choice of the orbital sub-space, the consequences of structural changes onto the localization of Wannier functions, and the changes related to the strength of screening processes.

\section{The choice of the orbital sub-space}

The construction of an effective low energy system depends very much upon the choice of the orbitals that it consists of. In particular, the dimension of the orbital sub-space $\mathcal{H}^{\text {low }}$ has a strong impact on the localization of the Wannier functions. Indeed, from the maximally localized Wannier function point of view, a larger subspace will allow, for a given orbital, for the mixing in of more Kohn-Sham orbitals. Therewith, the variational freedom to localize the basis functions becomes larger, and the interaction elements bigger. See, for an example, Ref. ${ }^{15}$. We thus expect the interaction matrix elements of the pd-model to be larger than in the d-only model. 


\section{Wannier functions under pressure}

External pressure, or structural changes in general, provide another impetus to alter not only the one-particle band-structure, but also the Wannier functions and thus the bare Coulomb interaction matrix elements.

The fact that Wannier functions are not eigenfunctions even of the non-interacting problem, may result in counter-intuitive tendencies. In Ref. ${ }^{14}$, we established generic behaviors of maximally localized Wannier functions and interaction matrix elements under pressure. In order to put things into perspective, we shall here briefly summarize some findings of this work :

As a model system, we consider a tight-binding parametrization of a one dimensional solid, in which case Wannier functions are already maximally localized if inversion symmetry is verified, ${ }^{11,62}$ and limit the discussion to the case of a single band. Details can be found in Ref. ${ }^{14}$. As building blocks of the basis functions we take hydrogen-like 1 s orbitals in one dimension, $\chi(x)=1 / \sqrt{a_{0}} \exp \left(-|x| / a_{0}\right)$, with the Bohr radius $a_{0}$. The Bloch eigenfunction is given by $\psi_{k}(x)=$ $A_{k} \sum_{R} e^{\imath k R} \chi(x-R)$, where $A_{k}$ assures the normalization. The maximally localized Wannier functions are then $\psi_{R}(x)=\sum_{n} A_{n} \chi(x-n a-R)$, with the lattice constant $a$, while $A_{n}$ is a Fourier transform of $A_{k}$ and also incorporates the normalization of the Wannier function. In the limit of large atomic separation $\left(a \gg a_{0}\right)$, the Wannier functions will equal the atomic orbitals. Thus $A_{n}=\delta_{n, 0}$ for the representative site " 0 ". When pressure is applied, and the lattice constant shrinks, overlaps of the (non-orthogonal) atomic orbitals causes contributions from neighboring sites to mix into the basis functions, and the distribution $A_{n}$ broadens. This leads to an increase in the (nearest neighbor) transfer integral $t$ and a concomitant increase of the spread (as defined $i^{11}$ ). In this sense, charge carriers become more delocalized, as expected. The tails of the Wannier functions extent over several lattice constants, before the originally exponential decay sets in. However, as can be shown, ${ }^{14}$ the coefficient $A_{0}$ in the above decomposition on the array of atomic orbitals, i.e. the strength of the on-site orbital, grows, and thus becomes larger than one. This results in a greater probability density $\left|\psi_{R}(x)\right|^{2}$ around the site origins, $x-R=0$, when pressure is applied. This in turn causes a larger local Coulomb interaction matrix element $V$, Eq. (2) . Hence, the pressure induced delocalization is accompanied by a larger local interaction, whereas, intuitively, one might have expected the opposite. In our one band toy-model, since $P_{r}=0$ (see Eq. (3)) the screened Coulomb interaction $U$ equals the bare Coulomb interaction $V$. Moreover it is maximal in the maximally localized Wannier basis. ${ }^{14}$

\begin{tabular}{r||c|c|c|c}
$\mathrm{P}$ [GPa] & $t_{p-t_{2 g}}$ & $\Delta_{c t}$ & $t_{p-t_{2 g}}^{2} / \Delta_{c t}$ & $t_{t_{2 g}-t_{2 g}}$ \\
\hline \hline $0(\mathrm{~dB} 1)$ & 0.60 & 3.5 & 0.10 & 0.21 \\
$100(\mathrm{~dB} 1)$ & 0.83 & 5.5 & 0.13 & 0.20 \\
100 (B1) & 0.91 & 6.5 & 0.13 & 0.41
\end{tabular}

TABLE II: Comparison between the direct and oxygen mediated contributions to the effective transfer element of the occupied $t_{2 g}$ orbitals for the $\mathrm{B} 1 / \mathrm{dB} 1$ phase of $\mathrm{MnO} . \Delta_{c t}=$ $\bar{\epsilon}_{t_{2 g}}^{o c c}-\bar{\epsilon}_{2 p}$ is the charge transfer energy. Shown are the largest transfers for nearest neighbors of same spin. All energies in $\mathrm{eV}$.

\section{Screening strength and the interaction.}

While the above changes in overlaps and hybridizations directly enter the calculation by modifying the Wannier functions, that influence the matrix elements of any quantity, pressure moreover enters the construction of the effective low energy system by means of the bandstructure. Indeed, according to Eq. (3), pressure induced modifications in the one-particle excitations will alter the screening strengths of possible transitions. When bandwidths, crystal fields or bonding/antibonding splittings get enhanced upon the compression of the system, the screening will become less effective and, therewith, the Hubbard $U$ larger. Besides this energetic effect, evident from the denominators in Eq. (3), there is also an effect of matrix elements when expressing the polarization in the Wannier basis. For an example of the interplay of both effects see Ref. ${ }^{14}$. Contrary to the preceding section, the transition energies and the matrix elements affect not the bare Coulomb interaction, but only screened quantities.

\section{RESULTS AND DISCUSSION}

\section{A. $\mathrm{MnO}$ in the low pressure regime : The antiferromagnetic insulating dB1 phase}

\section{1. bandstructure}

Fig. 1 displays the LSDA band-structure of antiferromagnetic $\mathrm{MnO}$ for different pressures and structures. Explicitly marked are also the bands constructed from the Wannier orbitals that are used for the construction of the effective "pd" low energy system (the bands of the effective "d-only" model achieve the same precision). Using the maximally localized Wannier function formalism for entangled bands, ${ }^{12}$ the choice of Hilbert space for the $\mathrm{Mn} 3 d$ and $\mathrm{O} 2 p$ orbitals results in a clean distinction from the $\mathrm{Mn} 4 \mathrm{~s}$ band that crosses the d-bands.

The corresponding LSDA densities of states (DOS) of the pd-model are shown in Fig. 2. As we can see, pressure induces an increase in the crystal-field splitting, $\Delta_{c f}$, i.e. a larger separation between the $e_{g}$ and $t_{2 g}$ KohnSham excitations (both the occupied and unoccupied), to an extent that the moving of the $e_{g}$ weight towards 


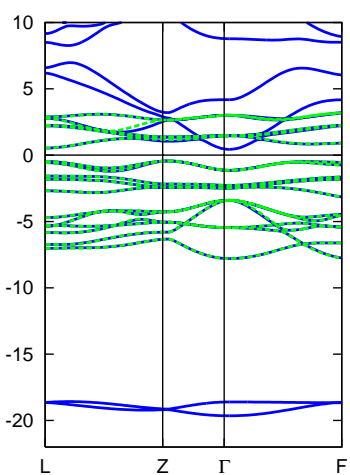

(a) $0 \mathrm{GPa}-\mathrm{B} 1(\mathrm{NaCl})$

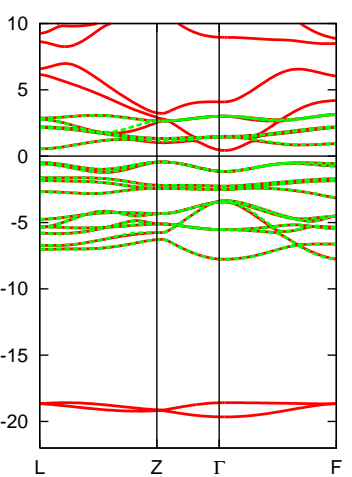

(b) $0 \mathrm{GPa}-\mathrm{dB} 1$

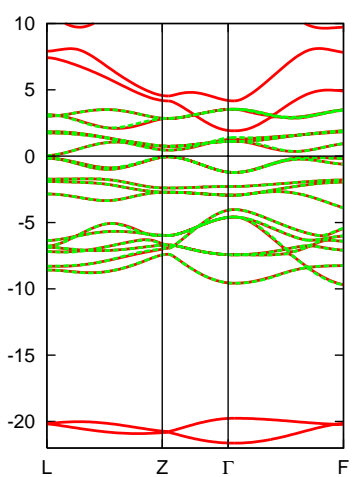

(c) $52.5 \mathrm{GPa}-\mathrm{dB} 1$

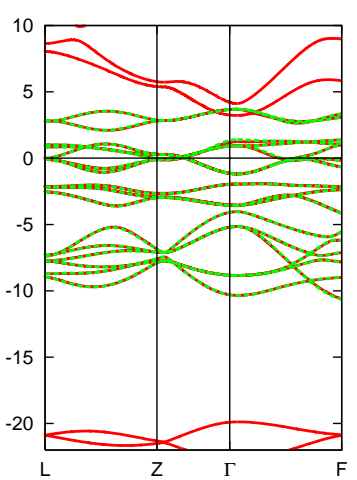

(d) 100GPa - dB1

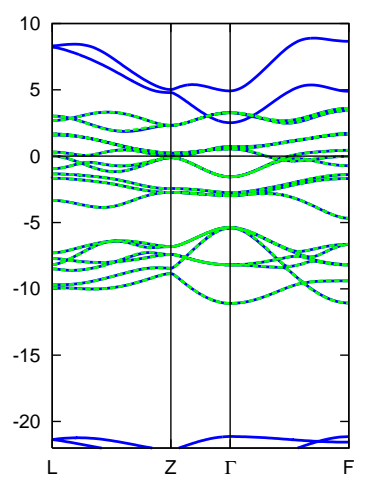

(e) $100 \mathrm{GPa}-\mathrm{B} 1(\mathrm{NaCl})$

FIG. 1: (color online) LDA band-structure of antiferromagnetic B1/dB1 MnO. Shown are the Kohn-Sham bands (solid lines) and the eigenvalues of the Wannier Hamiltonian (dashed). The vertical axis is energy (in eV) with the Fermi level at the origin. While (b) - (d) correspond to the true distorted dB1 structure at different pressures, (a) \& (d) show the band-structure for the artificial $\mathrm{B} 1(\mathrm{NaCl})$ structure for the indicated pressures.

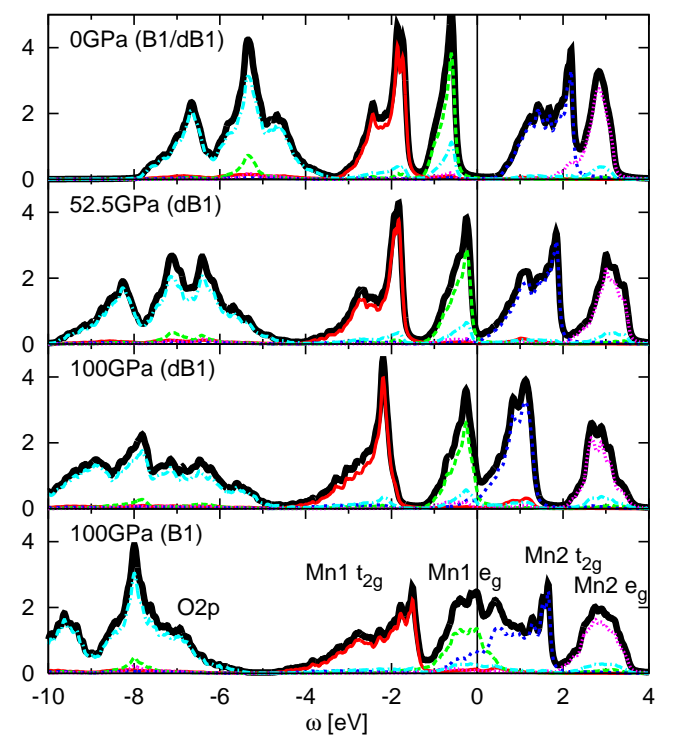

FIG. 2: (Color online) Density of states (DOS) (in $1 / \mathrm{eV}$ ) of antiferromagnetic $\mathrm{B} 1 / \mathrm{dB} 1 \mathrm{MnO}$ for increasing pressure (top to bottom). Shown are the site-summed contributions of one spin component of the pd-model. The lowest panel displays fictitious $\mathrm{B} 1 \mathrm{MnO}$ at $100 \mathrm{GPa}$ at the experimental volume. Different partial DOS are depicted as indicated in the graph at the bottom. For $P=0 \mathrm{GPa}$, the DOS of the $\mathrm{dB} 1$ and the B1 phase do not differ significantly on the shown scale.

the Fermi level eventually causes the system to become metallic. ${ }^{40}$ Concomitant with the increase in crystalfield, pressure results in a growing $\mathrm{Mn}-\mathrm{O}$ hybridization $t_{p d}=\left\langle p \mathbf{0}\left|H^{\mathrm{KS}}\right| d \mathbf{0}\right\rangle$, where $H^{\mathrm{KS}}$ is the Kohn-Sham Hamiltonian. This effect is especially large for the $e_{g}$ orbitals, as is clear from the octahedral coordination. For values, see the later discussed Tab. II (occupied $t_{2 g}$ orbitals), and III ( $e_{g}$ orbitals). Most notably is the fact, that the partial d-bandwidths do not increase with pressure. The origin of this is identified when comparing results for the experimental dB1 phase with the undistorted $\mathrm{B} 1(\mathrm{NaCl})$ structure of the same volume. As evident from Fig. 1 (d,e), and Fig. 2, the omission of the rhombohedral distortion causes the bandwidths to substantially increase. Indeed, in fictitious $\mathrm{B} 1 \mathrm{MnO}$, the closing of the gap can be mainly attributed to the change in bandwidth, as was stated in Ref. ${ }^{19,63}$ where the distortion was neglected. The evolution of the bandwidths can be quantified by looking at the effective Mn3d nearest neighbor transfer elements for a given spin. The latter consists of two major contributions : the direct hopping $t_{d d}=\left\langle d \mathbf{0}\left|H^{\mathrm{KS}}\right| d \mathbf{R}\right\rangle$ ( $\mathbf{R}$ nearest neighbor unit-cell of $\mathbf{0}$ ), and the oxygen mediated transfer $t_{p d}^{2} / \Delta_{c t}$, with the hybridization $t_{p d}$, and the charge transfer energy $\Delta_{c t}$. The (largest) nearest (spin) neighbor transfers of the occupied $t_{2 g}$ orbitals, $t_{t_{2 g}-t_{2 g}}$, the corresponding hybridization with the oxygen $2 p$ orbitals, $t_{p-t_{2 g}}$, and the charge transfer energy $\Delta_{c t}=\bar{\epsilon}_{t_{2 g}}^{o c c}-\bar{\epsilon}_{2 p}$ as measured by the band centers ${ }^{73}$, are collected in Tab. II for ambient pressure and 100GPa, for both, the experimental $\mathrm{dB} 1$ and the undistorted B1 structure.

As a result of the joint increase of $t_{p d}$ and $\Delta_{c t}$, the oxygen mediated contribution to the $t_{2 g}$ bandwidth remains roughly of the same magnitude, irrespective of pressure and distortion. The direct $t_{2 g}$-transfer for the distorted dB1 structure is also constant with pressure. However, it is substantially structure dependent. Indeed the nearest neighbor $t_{2 g}-t_{2 g}$ transfer doubles when the distortion is neglected, causing the effective hopping to augment by $64 \%$, demonstrating the critical need to include the distortion for a proper description of $\mathrm{dB} 1 \mathrm{MnO}$ under pressure. As to the unoccupied $t_{2 g}$ orbitals, the transfers and energy differences are larger, but the same argument 


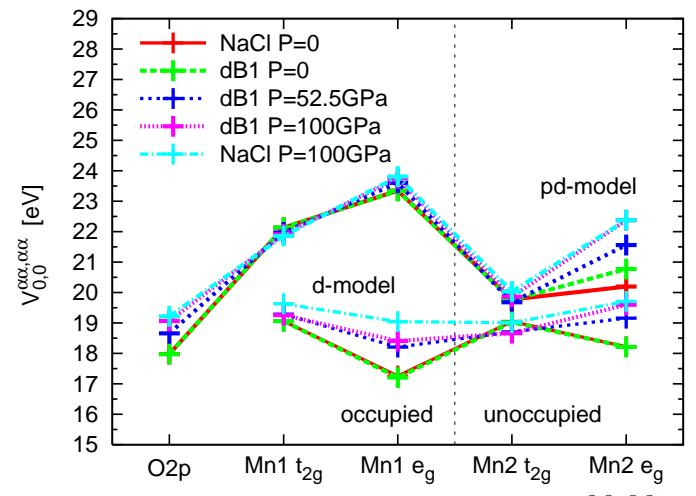

(a) on-site bare Coulomb interaction $V_{\mathbf{0}, \mathbf{0}}^{\alpha \alpha, \alpha \alpha}$

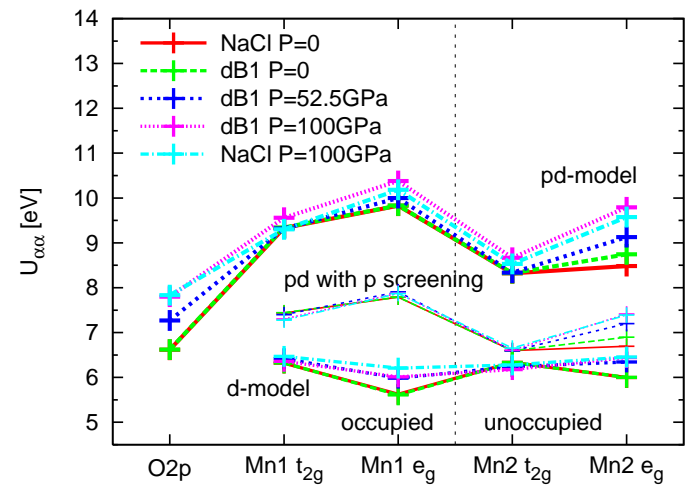

(b) partially screened interaction $U_{\alpha \alpha}=W_{r \mathbf{0}, \mathbf{0}}^{\alpha \alpha \alpha}(\omega=0)$

FIG. 3: (Color online) local Coulomb interaction of the antiferromagnetic dB1 phase of MnO for different pressures. (a) diagonal elements of the local, bare interaction $V$ for the pd and d-only model, and resolved for the different orbitals $\alpha$. (b) zero frequency limit of the RPA partially screened local interaction $U_{\alpha \alpha}=W_{r_{\mathbf{0}} \mathbf{0}}^{\alpha \alpha, \alpha \alpha}(\omega=0)$ for the pd model (with and without p screening), and the d-only model. For comparison are shown also the results of undistorted, $\mathrm{B} 1$ ( $\mathrm{NaCl}$ ) structured $\mathrm{MnO}$.

as above holds, for the trends are the same. We conclude that within LDA (in contrast to previous works ${ }^{19}$ (see also the recent ${ }^{63}$ ) relying on the undistorted structure), the gap closure under pressure is propelled by the change in crystal field and not by a broadening of band-widths.

\begin{tabular}{r||c|c|c|c}
$\mathrm{P}$ [GPa] & $t_{p-e_{g}}^{\text {unocc(occ) }}$ & $\widetilde{\Delta}_{c t}^{\text {unocc }(o c c)}$ & $t_{p-e_{g}}^{2} / \widetilde{\Delta}_{c t}$ & $t_{e_{g}-e_{g}}^{\text {unocc(occ) }}$ \\
\hline \hline $0(\mathrm{~dB} 1)$ & $1.24(1.08)$ & $8.0(4.75)$ & $0.19(0.25)$ & $0.05(0.06)$ \\
$100(\mathrm{~dB} 1)$ & $1.72(1.50)$ & $10.5(7.50)$ & $0.28(0.30)$ & $0.13(0.11)$ \\
100 (B1) & $1.82(1.63)$ & $10.5(7.50)$ & $0.32(0.35)$ & $0.14(0.13)$
\end{tabular}

TABLE III: Comparison between the direct and oxygen mediated contributions to the effective transfer elements of the unoccupied (occupied) $e_{g}$ orbitals for the B1/dB1 phase of Mn. $\widetilde{\Delta}_{c t}=\bar{\epsilon}_{e_{g}}-\bar{\epsilon}_{2 p}$ is the charge transfer energy. Shown are the largest transfers for nearest neighbors with the same spin. All energies in $\mathrm{eV}$.

The relevant quantities for the unoccupied (occupied) $e_{g}$ orbitals are compiled in Table III. For the occupied $e_{g}$ orbitals the situation is opposite to the $t_{2 g}$ : While the oxygen mediated contribution to the band-width, $t_{p-e_{g}}^{2} / \widetilde{\Delta}_{c t}^{o c c}$, at $100 \mathrm{GPa}$ depends sizeably on the structure $(\mathrm{B} 1 / \mathrm{dB} 1)$, the direct hopping is rather insensitive in this respect. For the unoccupied $e_{g}$ none of the values at $100 \mathrm{GPa}$ depends much on the structure, as can be inferred also from the similar shapes of the density of states. Yet, overall the effective bandwidths of the dB1 phase are always a little smaller than for the undistorted phase. Thus, the rhombohedral distortion is effectively reducing all partial d-bandwidths.

\section{The Coulomb interaction}

a. The bare interaction. As explained in a model context in Ref. ${ }^{14}$, changes in the orbital overlaps and hybridizations are expected to have an impact on the bare Coulomb interaction matrix $V$ of Eq. (2). The on-site elements of the latter for antiferromagnetic dB1 $\mathrm{MnO}$ are shown in Fig. 3(a), both for a pd and a d-only Wannier basis set, and for different pressures.

Owing to the distortion axis, the degeneracy properties of the Mn3d orbitals do not change with pressure. Moreover, in the current case, the maximally localized Wannier functions retain the degeneracy of the LMTOs, and while the LDA wavefunctions were merely the initial guesses for the maximally localized Wannier functions, we keep the labeling of " $t_{2 g}$ ", " $e_{g}$ ", " $d_{x y}$ " and alike. In some cases, especially for entangled bands, the localization procedure might cause the resulting Wannier functions to acquire a different symmetry.

In the case of the pd-model, the matrix elements are sensitive to pressure only for the $\mathrm{O} 2 p$ and the unoccupied $e_{g}$ orbitals. There, the changes in the interaction are quite significant and reach up to $10 \%$. This is a natural consequence of our above remarks : It is precisely those orbitals whose bondings changes the most under pressure, namely the hybridizations between them increase considerably. As summarized in the Method section, and discussed in detail in Ref. ${ }^{14}$, this allows the respective Wannier functions to accumulate more weight at small distance, while an overall increase in spread accounts for the gain in hybridization. This is also the reason why, for the pd-model, the $e_{g}$ elements are larger than the $t_{2} g$ ones : the larger hybridizations of the $e_{g}$ orbitals with the oxygen $2 p$ ones, allow the corresponding maximally localized Wannier functions to reduce their extension.

As anticipated, the elements of the d-model are lower 


\begin{tabular}{|c|c|c|}
\hline $\mathrm{P}[\mathrm{GPa}]$ & $U_{p d}[\mathrm{eV}]$ & $U_{d d}^{n n}[\mathrm{eV}$ \\
\hline $0.0(\mathrm{~dB} 1)$ & 2.25 & 1.51 \\
\hline $52.5(\mathrm{~dB} 1)$ & 2.44 & 1.69 \\
\hline $100.0(\mathrm{~dB} 1)$ & 2.65 & 1.96 \\
\hline $100.0 \quad(\mathrm{~B} 1)$ & 2.61 & 1.74 \\
\hline $96.5 \quad(\mathrm{~B} 8)$ & 1.66 & 1.11 \\
\hline $120.0 \quad(\mathrm{~B} 8)$ & 1.86 & 1.26 \\
\hline $160.0 \quad(\mathrm{~B} 8)$ & 1.98 & 1.42 \\
\hline
\end{tabular}

TABLE IV: Averaged nearest neighbor inter-atomic interactions $U_{p d}=W_{r \mathbf{0}, \mathbf{0}}^{p p, d d}(\omega=0)$ and $U_{d d}^{n n}=W_{r_{\mathbf{0}}, \mathbf{0}}^{d_{1} d_{1}, d_{2} d_{2}}(\omega=0)$ (where $d_{1 / 2}$ is a short hand for the d-orbitals of the 1./2. Mn atom in the cell) for the pd-model of the B1/dB1 phase (top) and the B8 phase (bottom) for different pressures.

than for the pd case, since the Wannier functions are more extended. Having restrained the variational freedom to the $\mathrm{d}$ orbitals, their matrix elements acquire a larger dependence on pressure than in the pd case. This is a result of the fact that the intra-d hoppings, especially the $e_{g}$ ones, must make up for the dispersion caused by oxygen mediated transfers that are not present in the smaller basis.

b. The partially screened interaction. While the bare Coulomb interaction was mainly a mean to analyze the pressure dependence of the Wannier functions, the quantity that is needed for the formulation of the effective models is the partially screened Coulomb interaction. In Fig. 3 (b) we show, for the same pressures as before, the Hubbard $U$ for the three different types of models, as introduced in the Method's section : the full pd-model, the pd-model with pd-screening (for a treatment in which only the d-orbitals will be supplemented by a local interaction), and the d-only model. The magnitudes of the corresponding interactions decrease in the given order : The interactions in the pd-model are smaller when the p-screening is present, and the interactions in the d-only model are yet smaller because of the larger extension of the Wannier functions. The pressure dependence of the elements has the same tendencies as the bare Coulomb interactions. Yet, we note that the relative difference, $\left(U^{100 G P a}-U^{0 G P a}\right) / U^{0 G P a}$, is larger for the Hubbard $U$ than for $V$, the pressure influence thus stronger. This is understood from a decrease in screening strength owing to the larger separation in energy between occupied and unoccupied states as pressure increases (see e.g. Ref. ${ }^{14}$ for a discussion), as apparent from the band-structure, Fig. 2. On an absolute scale, and for the given pressure range, the Hubbard $U$ changes by up to $1 \mathrm{eV}$ for both the Mn3d and the O2p orbitals - a non-negligible effect given the aforementioned modifications in the magnitude of the transfer integrals.

For the sake of completeness and to put the values further into perspective, Table IV (upper part) contains the nearest neighbor partially screened interactions $U_{p d}=W_{r_{\mathbf{0}, 0}}^{p p, d d}(\omega=0)$ and $U_{d d}^{n n}=W_{r_{\mathbf{0}, \mathbf{0}}}^{d_{1} d_{1}, d_{2} d_{2}}(\omega=0)$ (where $d_{1 / 2}$ is a short hand for the d-orbitals of the 1./2. Mn atom in the cell) within the pd-model and averaged over the respective orbital subset. Both elements are roughly a factor of four smaller than the intra-atomic interactions.

\begin{tabular}{r||c|c|c|c|c}
$0(100) \mathrm{GPa}$ & $d_{x y}$ & $d_{x z}$ & $d_{z^{2}}$ & $d_{y z}$ & $d_{x^{2}-y^{2}}$ \\
\hline \hline$d_{x y}$ & - & $0.61(0.62)$ & $0.66(0.68)$ & $0.61(0.62)$ & $0.38(0.37)$ \\
$d_{x z}$ & $0.61(0.62)$ & - & $0.46(0.45)$ & $0.61(0.62)$ & $0.59(0.60)$ \\
$d_{z^{2}}$ & $0.66(0.68)$ & $0.46(0.45)$ & - & $0.45(0.45)$ & $0.58(0.65)$ \\
$d_{y z}$ & $0.61(0.62)$ & $0.61(0.62)$ & $0.45(0.45)$ & - & $0.59(0.60)$ \\
$d_{x^{2}-y^{2}}$ & $0.38(0.37)$ & $0.59(0.60)$ & $0.58(0.65)$ & $0.59(0.60)$ & -
\end{tabular}

TABLE V: Hund's coupling $J=W_{r_{\mathbf{0}, 0}}^{\alpha \beta, \beta \alpha}(\omega=0, \alpha \neq \beta)$ of antiferromagnetic dB1 MnO and the d-only model for the extreme pressures 0 and 100GPa.

As a function of pressure, both $U_{p d}$ and $U_{d d}^{n n}$ increase. As was true for the diagonal elements of the Hubbard U, the pressure dependence of these off-diagonal interactions is a feature mostly intrinsic to the Wannier functions and not the screening strength.

The Hund's coupling, $J=W_{r_{\mathbf{0}, \mathbf{0}}}^{\alpha \beta, \beta \alpha}(\omega=0, \alpha \neq \beta)$, is compiled below in Table $\mathrm{V}$. While being largely anisotropic with respect to the orbital (for the Mn d orbitals, values range from $0.4 \mathrm{eV}$ to $0.7 \mathrm{eV}$ at ambient pressure), it does not depend significantly on pressure. 


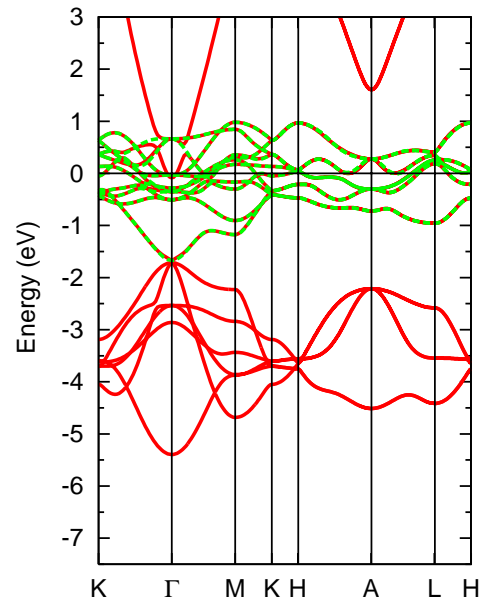

(a) $96.5 \mathrm{GPa}-\mathrm{B} 8$

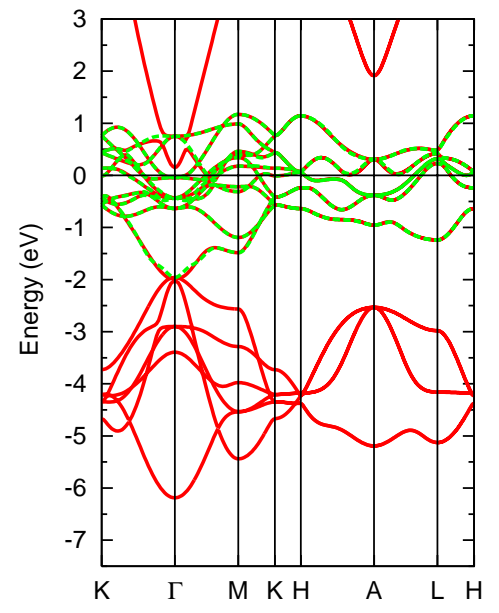

(b) $120 \mathrm{GPa}-\mathrm{B} 8$

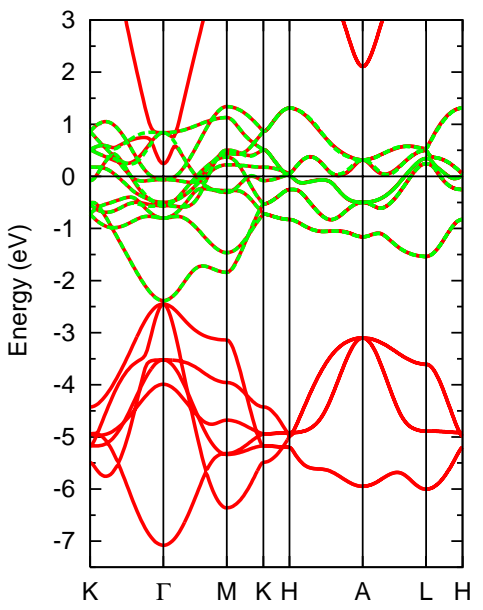

(c) $160 \mathrm{GPa}-\mathrm{B} 8$

FIG. 4: (color online) LSDA band-structure of paramagnetic MnO in the B8 high pressure phase. Shown are the Kohn-Sham bands (solid) and the eigenvalues of the Wannier Hamiltonian (dashed). The vertical axis is energy (in eV) with the Fermi level at the origin.

\section{B. $\mathrm{MnO}$ at high pressure : the paramagnetic $\mathrm{B} 8$ phase}

\section{The band-structure}

The $\mathrm{B} 8$ phase of $\mathrm{MnO}$ is of NiAs (B8) structure. As discussed in Ref. ${ }^{26}$, the $\mathrm{B} 8$ and $\mathrm{dB} 1$ structure are rather similar, and a transition from the high pressure dB1 to the B8 phase requires a moving of oxygen atoms from "distorted octahedral" to "perfect trigonal" positions.

Yet, in the pressure dependence, there is an important difference : Augmenting pressure in the dB1 phase leads to an increase of the trigonal angle, and thus to anisotropic changes. This results in an almost pressureinsensitive bandwidths, as discussed above. In the B8 phase, the c/a ratio basically remains unchanged, and it is thus only the uniform modification of the lattice constant that accounts for the change in volume. We note that the different coordination causes the d-orbitals to split into two doubly degenerate $e_{g}^{1,2}$ and a single $a_{g}$ orbitals (see e.g. Ref. ${ }^{42}$ ). Fig. 4 shows the band-structure of $\mathrm{B} 8 \mathrm{MnO}$ for various pressures, along with the bands in the Wannier gauge for the d-model. The corresponding DOS is displayed in Fig. 5. The lowest indicated pressure is in fact smaller than the largest value we had chosen for the dB1 phase. This is justified by the fact that both phases are coexisting over a finite pressure range, as evidenced from optics ${ }^{37,38}$ and x-ray experiments. ${ }^{26}$

Of course, the LDA yields a metal for all pressures. Since the Mott transition within the paramagnetic B8 phase only takes place at finite temperatures, this is congruent with experiments. However, we used again experimental structures and volumes at ambient temperature, ${ }^{26}$ since our aim is the construction of a model for the metal-insulator transition within the B8 phase. Continuing our remarks from the Methods section, this model construction is approximate. While it is perfectly admissible that the one-particle dispersion is metallic, one might object that the pd screening that we include in the determination of the Hubbard $U$ could be overestimated since the exact Green's function will have excitations with a larger separation because of the insulating nature of the system.

In the LDA band-structure, Fig. 4, the d-bandwidth becomes slightly larger with increasing pressure. As mentioned in Ref. ${ }^{54}$ this restricted increase is a result of the fact that the d-bandwidth is mainly constituted by the hybridization contribution of the $\mathrm{O} 2 p$ orbitals. Yet, while $t_{p d}$ increases with pressure, so does the charge transfer energy $\Delta_{c t}$, and thus the effective hopping, $t_{p d}^{2} / \Delta_{c t}$, does not increase much (cf. our discussion of the dB1 phase.). The preponderant effect of pressure on the bandstructure is a moving away from the Fermi surface of the occupied $\mathrm{O} 2 p$ and the unoccupied $\mathrm{Mn} 4 s, 4 p$ and $4 d$ orbitals, owing to greater hybridization splittings.

\section{The interaction}

Given the above changes in bandwidths, the bare Coulomb interaction is expected to be roughly pressure independent. As can be inferred from Fig. 6 (a), which displays this quantity for both the pd and the d-only model, this is found indeed to be the case. The absolute values are comparable in size to those of the $\mathrm{dB} 1$ phase.

The pressure dependence is decisively different for the partially screened interaction -the Hubbard U- shown in Fig. 6, as above, again for the three different types of models. As pressure augments, the changes induced 


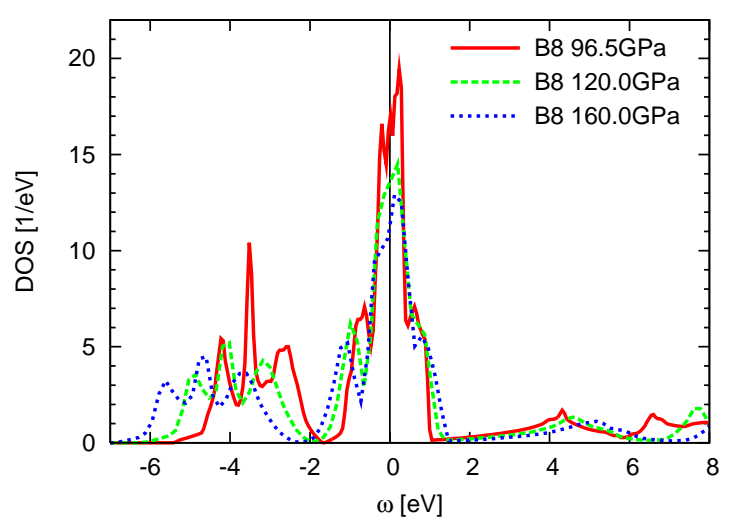

FIG. 5: (Color online) LDA density of states (DOS) of the paramagnetic $\mathrm{B} 8$ phase of $\mathrm{MnO}$ for various pressures. The Fermi level corresponds to $\omega=0$.

to the bands-structure lead to a decrease in screening strength, resulting in an almost isotropic increase of the effective interaction of up to about $0.1 \mathrm{eV} / 10 \mathrm{GPa}$ for the pd-model.

The average nearest neighbor interaction elements $U_{p d}$ and $U_{d d}^{n n}$ (for definitions see above) for the pd-model are compiled in Tab. IV. The trend with pressure is the same as for the intra-atomic elements, i.e. they grow with pressure. However, like in the dB1 phase, they are smaller by a factor of about four with respect to the intra-atomic elements. While the pressure dependence of the diagonal terms of the screened interaction could be attributed almost exclusively to changes in the screening, it is notable that the off-diagonal elements of already the bare Coulomb matrix elements change by almost 10\% from the lowest to the highest pressure considered.

What had been said about the Hund's coupling of the dB1 phase is also true here, as seen from Table VI. Being less susceptible to screening processes, it does not show a significant pressure dependence. However, it is, again, sensitive to the combination of orbitals involved, and values can vary by as much as $65 \%$. Since the physics of the high spin to low spin transition ${ }^{54}$ within the B8 phase is sensitive not to the diagonal elements of the interactions (which change considerably), but mostly to changes in $\mathrm{J}^{74}$ (that are small), arguments made about the transition mechanism ${ }^{54}$ may not be affected when using the pressure dependent matrix elements instead of constant ones. Yet, spectra will change on a quantitative level.

\begin{tabular}{r||c|c|c|c|c}
$96(160) \mathrm{GPa}$ & $d_{x y}$ & $d_{x z}$ & $d_{z^{2}}$ & $d_{y z}$ & $d_{x^{2}-y^{2}}$ \\
\hline \hline$d_{x y}$ & - & $0.49(0.51)$ & $0.70(0.69)$ & $0.61(0.61)$ & $0.58(0.54)$ \\
$d_{x z}$ & $0.49(0.51)$ & - & $0.48(0.46)$ & $0.63(0.65)$ & $0.62(0.63)$ \\
$d_{z^{2}}$ & $0.70(0.69)$ & $0.48(0.46)$ & - & $0.48(0.49)$ & $0.69(0.69)$ \\
$d_{y z}$ & $0.61(0.61)$ & $0.63(0.65)$ & $0.48(0.49)$ & - & $0.49(0.51)$ \\
$d_{x^{2}-y^{2}}$ & $0.58(0.54)$ & $0.62(0.63)$ & $0.69(0.69)$ & $0.49(0.51)$ & -
\end{tabular}

TABLE VI: Hund's coupling $J=W_{r_{\mathbf{0}, \mathbf{0}}}^{\alpha \beta, \beta \alpha}(\omega=0, \alpha \neq \beta)$ of paramagnetic B8 $\mathrm{MnO}$ for the d-only model and the pressures 96 and 160GPa.

\section{SUMMARY, CONCLUSIONS AND PERSPECTIVES}

In conclusion, we have studied the impact of external pressure onto the construction of effective low energy many-body models for the realistic example of manganese monoxide. This compound is particularly challenging for a first principle description, since energies, such as the transfer integrals, the Hubbard $U$ and the charge transfer energies are of the same magnitude. On the level of band-structures, we concluded that the pressure induced vanishing of the gap in the antiferromagnetic phase of $\mathrm{MnO}$ is driven by the crystal field rather than by an increase of band-widths, the latter of which is found to be limited by the growing rhombohedral distortion. The high pressure paramagnetic phase, on the contrary, exhibits the usual pressure caused increase of hybridizations and bandwidths. Using the constraint RPA approach within setups of maximally localized Wannier functions, we further found that, for a quantitative description, the pressure dependence of the Hubbard $U$ can not be neglected. While the pressure sensitivity in the low pressure antiferromagnetic phase of $\mathrm{MnO}$ is dominated by compression induced changes of the bare Coulomb interaction, the effective interaction of the high pressure paramagnetic phase is mostly determined by modifications in the band-structure that affect the screening properties of the system. Our ab initio constructed low energy models can provide a quantitative starting point for the application of sophisticated many-body methods. 


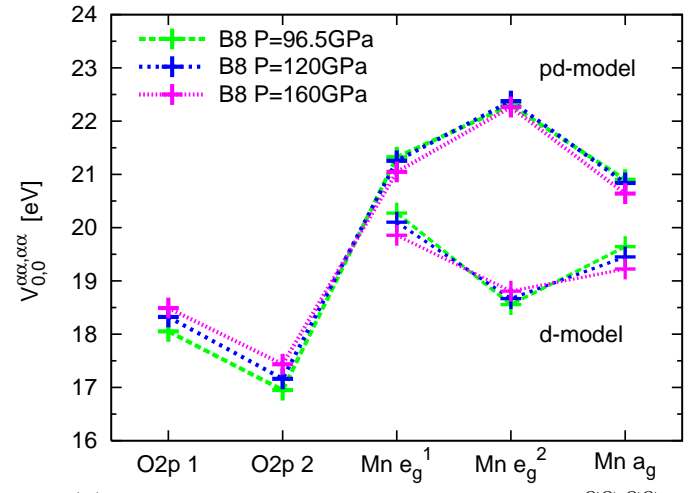

(a) on-site bare Coulomb interaction $V_{\mathbf{0}, \mathbf{0}}^{\alpha \alpha, \alpha \alpha}$

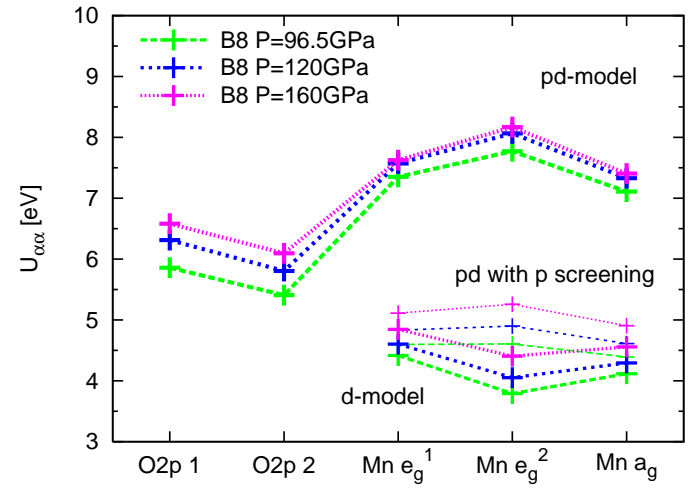

(b) partially screened interaction $U_{\alpha \alpha}=W_{r_{\mathbf{0}, \mathbf{0}}^{\alpha \alpha}}^{\alpha \alpha, \alpha}(\omega=0)$

FIG. 6: (Color online) local Coulomb interaction of the paramagnetic B8 phase of $\mathrm{MnO}$ for different pressures. (a) diagonal elements of the local, bare interaction $V_{\mathbf{0}, \mathbf{0}}^{\alpha \alpha, \alpha \alpha}$ for the pd and d-only model, and resolved for the different orbitals $\alpha$. (b) zero frequency limit of the RPA partially screened local interaction $U_{\alpha \alpha}=W_{r_{\mathbf{0}, \mathbf{0}}}^{\alpha \alpha, \alpha \alpha}(\omega=0)$ for the pd model (with and without $\mathrm{p}$ screening), and the d-only model.

\section{Acknowledgments}

We gratefully acknowledge discussions with and comments by R. Sakuma, J. Kuneš, K. Terakura and R. Co- hen. This work was in part supported by the G-COE program of $\operatorname{MEXT}(G-03)$ and the Next Generation Supercomputer Project, Nanoscience Program from MEXT, Japan.
1 W. Kohn. Nobel lecture: Electronic structure of matterwave functions and density functionals. Rev. Mod. Phys., 71(5):1253-1266, Oct 1999.

${ }^{2}$ R. O. Jones and O. Gunnarsson. The density functional formalism, its applications and prospects. Rev. Mod. Phys., 61(3):689-746, Jul 1989.

${ }^{3}$ G. Kotliar and D. Vollhardt. Strongly correlated materials: Insights from dynamical mean-field theory. Physics Today, 57(3):53, 2004.

${ }^{4}$ V. I. Anisimov and O. Gunnarsson. Density-functional calculation of effective coulomb interactions in metals. Phys. Rev. B, 43(10):7570-7574, Apr 1991.

5 F. Aryasetiawan, M. Imada, A. Georges, G. Kotliar, S. Biermann, and A. I. Lichtenstein. Frequencydependent local interactions and low-energy effective models from electronic structure calculations. Phys. Rev. B, 70(19):195104, Nov 2004.

${ }^{6}$ M. Imada, A. Fujimori, and Y. Tokura. Metal-insulator transitions. Rev. Mod. Phys., 70(4):1039-1263, Oct 1998.

7 V. I. Anisimov, J. Zaanen, and O. K. Andersen. Band theory and mott insulators: Hubbard $\mathrm{u}$ instead of stoner i. Phys. Rev. B, 44(3):943-954, Jul 1991.

8 Matteo Cococcioni and Stefano de Gironcoli. Linear response approach to the calculation of the effective interaction parameters in the $l d a+u$ method. Phys. Rev. B, 71(3):035105, Jan 2005.

9 Taku Tsuchiya, Renata M. Wentzcovitch, Cesar R. S. da Silva, and Stefano de Gironcoli. Spin transition in magnesiow[u-umlaut]stite in earth's lower mantle. Physical Review Letters, 96(19):198501, 2006.

10 Han Hsu, Koichiro Umemoto, Matteo Cococcioni, and Re- nata Wentzcovitch. First-principles study for low-spin lacoo[sub 3] with a structurally consistent hubbard u. Physical Review B (Condensed Matter and Materials Physics), 79(12):125124, 2009

11 Nicola Marzari and David Vanderbilt. Maximally localized generalized wannier functions for composite energy bands. Phys. Rev. B, 56(20):12847-12865, Nov 1997.

12 Ivo Souza, Nicola Marzari, and David Vanderbilt. Maximally localized wannier functions for entangled energy bands. Phys. Rev. B, 65(3):035109, Dec 2001.

13 Takashi Miyake and F. Aryasetiawan. Screened coulomb interaction in the maximally localized wannier basis. Phys. Rev. B, 77(8):085122, 2008.

14 Jan M. Tomczak, T. Miyake, R. Sakuma, and F. Aryasetiawan. Effective coulomb interactions in solids under pressure. Phys. Rev. B, 79(23):235133, 2009.

15 T. Miyake, L. Pourovskii, V. Vildosola, S. Biermann, and A. Georges. d- and f-orbital correlations in the refeaso compounds. J. Phys. Soc. Jpn., 77SC(Supplement C):99102, 2008.

16 Kazuma Nakamura, Ryotaro Arita, and Masatoshi Imada. $\mathrm{Ab}$ initio derivation of low-energy model for iron-based superconductors lafeaso and lafepo. J. Phys. Soc. Jpn., 77:093711, 2008.

17 K. Terakura, T. Oguchi, A. R. Williams, and J. Kübler. Band theory of insulating transition-metal monoxides: Band-structure calculations. Phys. Rev. B, 30(8):47344747, Oct 1984.

18 Zhong Fang, Igor V. Solovyev, Hideaki Sawada, and Kiyoyuki Terakura. First-principles study on electronic structures and phase stability of mno and feo under high pres- 
sure. Phys. Rev. B, 59(2):762-774, Jan 1999.

19 Ronald E. Cohen, I. I. Mazin, and Donald G. Isaak. Magnetic Collapse in Transition Metal Oxides at High Pressure: Implications for the Earth. Science, 275(5300):654657, 1997.

${ }^{20}$ C. Franchini, V. Bayer, R. Podloucky, J. Paier, and G. Kresse. Density functional theory study of mno by a hybrid functional approach. Phys. Rev. B, 72(4):045132, 2005.

21 C. Franchini, R. Podloucky, J. Paier, M. Marsman, and G. Kresse. Ground-state properties of multivalent manganese oxides: Density functional and hybrid density functional calculations. Phys. Rev. B, 75(19):195128, 2007.

${ }^{22}$ Wei-Bing Zhang, Yong-He Deng, Yu-Lin Hu, Ke-Li Han, and Bi-Yu Tang. Structural distortion of b1-structured mno and feo. Solid State Comm., 142:6, 2007.

23 Deepa Kasinathan, J. Kuneš, K. Koepernik, Cristian V. Diaconu, Richard L. Martin, Ionut D. Prodan, Gustavo E. Scuseria, Nicola Spaldin, L. Petit, T. C. Schulthess, and W. E. Pickett. Mott transition of mno under pressure: A comparison of correlated band theories. Phys. Rev. B, 74(19):195110, 2006.

24 Fabien Tran, Peter Blaha, Karlheinz Schwarz, and Pavel Novák. Hybrid exchange-correlation energy functionals for strongly correlated electrons: Applications to transitionmetal monoxides. Phys. Rev. B, 74(15):155108, 2006.

25 J. van Elp, R. H. Potze, H. Eskes, R. Berger, and G. A. Sawatzky. Electronic structure of mno. Phys. Rev. B, 44(4):1530-1537, Jul 1991.

26 C. S. Yoo, B. Maddox, J.-H. P. Klepeis, V. Iota, W. Evans, A. McMahan, M. Y. Hu, P. Chow, M. Somayazulu, D. Häusermann, R. T. Scalettar, and W. E. Pickett. Firstorder isostructural mott transition in highly compressed mno. Phys. Rev. Lett., 94(11):115502, 2005.

27 H. A. Kramers. L'interaction entre les atomes magntognes dans un cristal paramagntique. Physica, 1(1-6):182 - 192, 1934.

${ }^{28}$ P. W. Anderson. Antiferromagnetism. theory of superexchange interaction. Phys. Rev., 79(2):350-356, Jul 1950.

29 J Zaanen and G A Sawatzky. The electronic structure and superexchange interactions in transition-metal compounds. Can. J. Phys., 65:1262, 1987.

30 C. G. Shull and J. Samuel Smart. Detection of antiferromagnetism by neutron diffraction. Phys. Rev., 76(8):12561257, Oct 1949.

31 C. G. Shull, W. A. Strauser, and E. O. Wollan. Neutron diffraction by paramagnetic and antiferromagnetic substances. Phys. Rev., 83(2):333-345, Jul 1951.

${ }^{32}$ H. Shaked, J. Faber, and R. L. Hitterman. lowtemperature magnetic structure of mno: a high-resolution neutron-diffraction study. Phys. Rev. B, 38(16):1190111903, Dec 1988.

33 J. D. Fitz Gerald S. L. Webb, Ian Jackson. High-pressure elasticity, shear-mode softening and polymorphism in mno. Physics of The Earth and Planetary Interiors, 52(1-2):117, 1988.

${ }^{34}$ P. Burlet D. Bloch, C. Vettier. Phase transition in manganese oxide at high pressure. Phys. Lett. A, 75(4):301, 1980.

35 Yasuhiko Syono. Electronic phase transition of transitionmetal monoxides under very-high pressure. RIKEN Review, 27:72, April 2000.

36 Tadashi Kondo, Takehiko Yagi, Yasuhiko Syono, Yuichi Noguchi, Toshiyuki Atou, Takumi Kikegawa, and Osamu
Shimomura. Phase transitions of mno to 137 gpa. Journal of Applied Physics, 87(9):4153-4159, 2000.

37 M. Kobayashi. Infrared spectroscopy of pressure-induced metallization in semiconductors. physica status solidi (b), 223(1):55-64, 2001.

38 Yoshimi Mita, Daisuke Izaki, Michihiro Kobayashi, and Shoichi Endo. Pressure-induced metallization of mno. Phys. Rev. B, 71(10):100101, 2005.

39 J. R. Patterson, C. M. Aracne, D. D. Jackson, V. Malba, S. T. Weir, P. A. Baker, and Y. K. Vohra. Pressure-induced metallization of the mott insulator mno. Phys. Rev. B, 69(22):220101, Jun 2004.

40 Shuhei Ohnishi. A theory of the pressure-induced highspin-low-spin transition of transition-metal oxides. Physics of The Earth and Planetary Interiors, 17(2):130, 1978.

41 M. D. Towler, N. L. Allan, N. M. Harrison, V. R. Saunders, W. C. Mackrodt, and E. Aprà. Ab initio study of mno and nio. Phys. Rev. B, 50(8):5041-5054, Aug 1994.

42 Deepa Kasinathan, K Koepernik, and W E Pickett. Pressure-driven magnetic moment collapse in the ground state of mno. New Journal of Physics, 9(7):235, 2007.

43 A. Svane and O. Gunnarsson. Transition-metal oxides

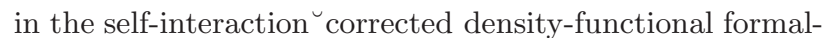
ism. Phys. Rev. Lett., 65(9):1148-1151, Aug 1990.

44 Z. Szotek, W. M. Temmerman, and H. Winter. Application of the self-interaction correction to transition-metal oxides. Phys. Rev. B, 47(7):4029-4032, Feb 1993.

45 Axel D. Becke. A new mixing of hartree-fock and local density-functional theories. The Journal of Chemical Physics, 98(2):1372-1377, 1993.

46 L. Hedin. New method for calculating the one-particle green's function with application to the electron-gas problem. Phys. Rev., 139(3A):A796-A823, Aug 1965.

47 F Aryasetiawan and O Gunnarsson. The gw method. Rep. Prog. Phys., 61(3):237-312, 1998.

48 Giovanni Onida, Lucia Reining, and Angel Rubio. Electronic excitations: density-functional versus many-body green's-function approaches. Rev. Mod. Phys., 74(2):601659, Jun 2002.

49 S. Massidda, A. Continenza, M. Posternak, and A. Baldereschi. Band-structure picture for mno reexplored: A model gw calculation. Phys. Rev. Lett., 74(12):23232326, March 1995.

50 Sergey V. Faleev, Mark van Schilfgaarde, and Takao Kotani. All-electron self-consistent $g w$ approximation: Application to si, mno, and nio. Phys. Rev. Lett., 93(12):126406, Sep 2004.

51 G. Kotliar, S. Y. Savrasov, K. Haule, V. S. Oudovenko, O. Parcollet, and C. A. Marianetti. Electronic structure calculations with dynamical mean-field theory. Rev. Mod. Phys., 78(3):865-951, Aug 2006.

52 A. Georges, G. Kotliar, W. Krauth, and M. J. Rozenberg. Dynamical mean-field theory of strongly correlated fermion systems and the limit of infinite dimensions. Rev. Mod. Phys., 68(1):13, Jan 1996.

53 S. Y. Savrasov and G. Kotliar. Linear response calculations of lattice dynamics in strongly correlated systems. Phys. Rev. Lett., 90(5):056401, Feb 2003.

54 Jan Kunes, Alexey V. Lukoyanov, Vladimir I. Anisimov, Richard T. Scalettar, and Warren E. Pickett. Collapse of magnetic moment drives the mott transition in mno. Nat Mater, 7(3):198, 2008.

55 J. Zaanen, G. A. Sawatzky, and J. W. Allen. Band gaps and electronic structure of transition-metal compounds. 
Phys. Rev. Lett., 55(4):418-421, Jul 1985.

56 A. Fujimori, N. Kimizuka, T. Akahane, T. Chiba, S. Kimura, F. Minami, K. Siratori, M. Taniguchi, S. Ogawa, and S. Suga. Electronic structure of mno. Phys. Rev. B, 42(12):7580-7586, Oct 1990.

57 G. Ghiringhelli, M. Matsubara, C. Dallera, F. Fracassi, A. Tagliaferri, N. B. Brookes, A. Kotani, and L. Braicovich. Resonant inelastic x-ray scattering of mno: L[sub 2,3] edge measurements and assessment of their interpretation. Phys. Rev. B, 73(3):035111, 2006.

58 M. Methfessel, Mark van Schilfgaarde, and R.I. Casali. A full-potential lmto method based on smooth hankel functions. in Electronic Structure and Physical Properties of Solids: The Uses of the LMTO Method, Lecture Notes in Physics. H. Dreysse, ed., 535:114-147, 2000.

${ }^{59}$ Gregory H. Wannier. Dynamics of band electrons in electric and magnetic fields. Rev. Mod. Phys., 34(4):645-655, Oct 1962.

${ }^{60}$ Clyde Edmiston and Klaus Ruedenberg. Localized atomic and molecular orbitals. Rev. Mod. Phys., 35(3):457-464, Jul 1963.

${ }^{61}$ F. Aryasetiawan, J. M. Tomczak, T. Miyake, and R. Sakuma. Downfolded self-energy of many-electron systems. Phys. Rev. Lett., 102(17):176402, May 2009. preprint: arXiv:0806.3373.

62 S. Kivelson. Wannier functions in one-dimensional disordered systems: Application to fractionally charged solitons. Phys. Rev. B, 26(8):4269-4277, Oct 1982.

${ }^{63}$ Luke Shulenburger, Sergey Y. Savrasov, and R. E. Cohen. Relative importance of crystal field versus bandwidth to the high pressure spin transition in transition metal monoxides. 2009. arXiv.org:0909.0325.

${ }^{64}$ L. V. Pourovskii, B. Amadon, S. Biermann, and A. Georges. Self-consistency over the charge density in dynamical mean-field theory: A linear muffin-tin implementation and some physical implications. Phys. Rev. B, 76(23):235101, 2007.

65 Takashi Miyake, Ferdi Aryasetiawan, and Masatoshi Imada. $\mathrm{Ab}$ initio procedure for constructing effective models of correlated materials with entangled band struc- ture. Physical Review B (Condensed Matter and Materials Physics), 80(15):155134, 2009.

66 S. Biermann, F. Aryasetiawan, and A. Georges. Firstprinciples approach to the electronic structure of strongly correlated systems: Combining the $g w$ approximation and dynamical mean-field theory. Phys. Rev. Lett., 90(8):086402, Feb 2003.

67 In the constrained LDA technique for the Hubbard $U$ of e.g. a d-manifold, the screening originating from transitions of other bands to and from the d-bands are eliminated, although they are not accountable for by the low energy system - a reason why the constraint LDA technique often overestimates the values of $U$.

68 This discards correlation induced changes in the charge density. See e.g. ${ }^{64}$ for a charge self-consistency scheme within DMFT.

69 Still, both LDA and RPA work at $T=0$.

70 In case the bands of the "low" and "high" subspace are entangled, the separation $P=P_{\text {low }}+P_{r}$ is not uniquely defined. For a way to overcome this arbitrariness see the recent ${ }^{65}$. Since in our case the entanglement is not too pronounced we do not employ this refinement.

71 While the RPA screening and thus also the effective interactions are energy dependent, so far, most many-body techniques only use static interactions, which is why we will discuss in this work only the low energy limit, i.e. for example the Hubbard $U_{\alpha \beta}=W_{r_{\mathbf{0}, 0}}^{\alpha \alpha \beta}(\omega=0)$ (using the conventions of Eq. (2)). For a discussion on the frequency dependence of the screened interaction see the Ref. ${ }^{5}$.

72 On a more general note, we stress, that the Wannier cRPA approach can be viewed as a first step towards the already mentioned combination of $\mathrm{GW}^{46-48}$ with the dynamical mean field theory (DMFT) ${ }^{52}$ "GW+DMFT", ${ }^{66}$ in which the Hubbard $U$ is promoted from a parameter to a quantity that obeys a self-consistency relation.

73 In this definition, it also includes the respective contributions from the exchange splitting.

74 J. Kuneš, private communication. 